ARTIGO

\title{
BEM-ESTAR, DESENVOLVIMENTO E ATIVIDADES ORGANIZADAS: UM ESTUDO COM ALUNOS ADOLESCENTES ${ }^{1}$
}

\author{
WELL-BEING, DEVELOPMENT AND ORGANIZED ACTIVITIES:
}

A STUDY WITH ADOLESCENTS STUDENTS

\author{
BIENESTAR, DESARROLLO Y ACTIVIDADES ORGANIZADAS: \\ UN ESTUDIO CON ALUMNOS ADOLESCENTES
}

\author{
Nuno Archer de Carvalho \\ Colégio Pedro Arrupe - Portugal
}

Feliciano Henriques Veiga

Instituto de Educação da Universidade de Lisboa - Portugal

\begin{abstract}
Resumo: Enquadramento: No estudo do Bem-estar psicológico e dos Recursos de desenvolvimento, destaca-se a participação dos adolescentes em atividades organizadas. Objetivo: Aprofundar a relação do Bem-estar psicológico e dos Recursos de desenvolvimento com a participação em atividades extracurriculares e de solidariedade. Metodologia: O estudo incluiu 201 alunos do $6^{\circ}, 9^{\circ}$ e $11^{\circ}$ ano de uma escola particular portuguesa e usou a Escala de Bem-Estar Psicológico para Adolescentes, o Developmental Assets Profile e três questões sobre a participação nas atividades. Resultados: Encontrou-se um número elevado de alunos que participam em atividades dos dois tipos. A participação diminui ao longo da adolescência. As correlações com o Bem-estar psicológico e os Recursos de desenvolvimento reforçam o potencial da participação nos dois tipos de atividade para o bem-estar e o desenvolvimento dos adolescentes. As diferentes correlações sugerem uma maior ligação da participação em atividades extracurriculares com a auto-estima, e entre a participação em atividades de solidariedade com a autorrealização, o crescimento pessoal e a resiliência. Conclusões: Valorização das oportunidades de participação em atividades dos dois tipos nos contextos educativos, com destaque para a escola. São apresentadas sugestões para estudos futuros.
\end{abstract}

Palavras chave: Adolescência; Atividades extracurriculares; Bem-estar psicológico.

Abstract: Context: The study of Psychological well-being and Developmental assets highlights adolescent participation in organized activities. Goal: To deepen the relation of Psychological wellbeing and Developmental assets with the participation in extracurricular and solidarity activities. Method: The study included 201 students from the 6th, 9th and 11th grades from a Portuguese private school and used the Psychological Well-being Scale for adolescents, the Developmental Assets Profile, and three questions about activity participation. Results: It was found that a high number of the students participate in both type of activities. This participation diminishes over the years. The correlations with Psychological well-being and Developmental assets strengthen the potential of the participation in both

\footnotetext{
${ }^{1}$ Este trabalho foi financiado por fundos nacionais através da FCT - Fundação para a Ciência e Tecnologia, IP.,
} no âmbito da Unidade de Investigação e Desenvolvimento em Educação e Formação UIDB/04107/2020. 
types of activities for adolescent well-being and development. Differences in correlations suggest a stronger connection between the participation in extracurricular activities and self-esteem, and between the participation in solidarity activities and self-realization, personal growth, and resilience. Conclusions: Valorisation of the opportunities for participation in the two types of activities in educational contexts, with emphasis to school settings. Suggestions for future studies are presented.

Keywords: Adolescence; Extracurricular activities; Psychological well-being.

Resumen: Antecedentes: En el estudio del Bienestar psicológico y de los Recursos de desarrollo se destaca la participación de lo adolescente en actividades organizadas. Objetivo: Profundizar la relación del Bienestar psicológico y de los Recursos de desarrollo con la participación en actividades extracurriculares y de solidaridad. Metodología: La muestra incluye 201 estudiantes de $6^{\circ}, 9^{\circ}$ y $11^{\circ}$ grado de una escuela particular. Se utiliza el Cuestionario de Bienestar Psicológico para evaluar el bienestar psicológico, el Developmental Assets Profile para evaluar los recursos de desarrollo y tres cuestiones para evaluar la participación en actividades. Resultados: Un número elevado de estudiantes participan en actividades de los dos tipos. La participación disminuí a lo largo de la adolescencia. Las correlaciones con el Bienestar psicológico y los Recursos de desarrollo refuerzan el potencial de la participación en los dos tipos de actividades en el bienestar y desarrollo adolescente. Diferencias en las correlaciones sugieren una mayor conexión entre la participación en actividades extracurriculares y la autoestima, y entre la participación en actividades de solidaridad y la autorrealización, lo crecimiento personal y la resiliencia. Conclusiones: Valorización de las oportunidades de participación en los dos tipos de actividades en los contextos educacionales, con destaque para la escuela. Sugerencias para nuevos estudios son presentadas.

Palabras clave: Adolescencia; Actividades extracurriculares; Bienestar psicológico.

\section{Introdução}

“O que eu quero principalmente é que vivam felizes!" É o desejo do poeta e professor Sebastião da Gama $(2003,23)$ para os seus alunos. E nós, o que desejamos para os nossos alunos? A missão atual da escola, para além da vocação académica, integra o bem-estar e o desenvolvimento integral de cada aluno (OECD, 2018), particularmente durante a adolescência, enquanto período-chave do desenvolvimento (Randall, Travers, Shapiro, \& Bohnert, 2016; Veiga, 2013; Veiga, García \& García 2019). Na demanda de uma melhor compreensão do que significa esta missão, destacam-se duas linhas de investigação que partilham a importância de ir além do défice para identificar e promover as capacidades, forças e possibilidades de cada contexto e de cada jovem (Benson, Scales, Hamilton \& Sesma, 2006).

A primeira linha de investigação é o Bem-estar Psicológico (BEP). O BEP surge como alternativa ao Bem-estar subjetivo, associado a uma visão hedonista da felicidade, assente na avaliação da frequência de emoções positivas e negativas e da satisfação com a vida (Freire, Zenhas, Tavares \& Iglésias, 2013). Carol Ryff (1989) critica a fragilidade conceptual e metodológica do bem-estar subjetivo, defende que o bem-estar deve ir para além das emoções e da satisfação com a vida e propõe uma visão do bem-estar que inclui as necessidades 
essenciais do funcionamento psicológico positivo e da saúde mental, conhecido por Bem-estar eudaimónico (Aristóteles), ou psicológico (BEP). O BEP enfatiza a questão do sentido, da autorrealização e da relação com os outros e inclui seis dimensões (Ryff, 2018a; 2018b): (1) Autonomia; (2) Domínio do meio; (3) Crescimento pessoal; (4) Relações positivas com outros; (5) Objetivos na vida; e (6) Aceitação de si.

A segunda linha de investigação é a dos Recursos de Desenvolvimento (RD) ou Developmental Assets e surge no contexto do Positive Youth Development (PYD) que tem na base a relação bidirecional entre os jovens e os seus contextos de desenvolvimento (Benson et al., 2006). Os RD são apresentados como nutrientes necessários ao desenvolvimento bemsucedido de todas as crianças e jovens, associados positivamente a indicadores de bem-estar e de desenvolvimento (Scales, Roehlkepartain \& Shramko, 2017) e associados negativamente a comportamentos de risco (Benson \& Scales, 2014). Os RD dividem-se em duas categorias: (a) os RD-Externos, que caracterizam os contextos de desenvolvimento; e (b) os RD-Internos, que caracterizam a criança ou jovem. Do trabalho de Peter Benson e do Search institute (Benson, Scales \& Syvertsen, 2011) nasce o referencial Developmental Assets Framework, constituído por $40 \mathrm{RD}$, distribuídos nas duas categorias apresentadas, cada uma das quais com quatro dimensões (Scales et al., 2017): (a) os RD-Externos que incluem as dimensões Suporte, Empoderamento, Limites e expectativas e Uso positivo do tempo, e (b) os RD-Internos que incluem as dimensões Compromisso com a aprendizagem, Valores positivos, Competências sociais e Identidade positiva.

Na revisão da literatura em torno do bem-estar e do desenvolvimento na adolescência é transversal o contributo das experiências e atividades fora do tempo de aulas a que os jovens têm acesso (Agans, Champine, Johnson, Erickson \& Yalin, 2015; Barber, Abbott, Neira \& Eccles, 2014; Vandell, Larson, Mahoney \& Watts, 2015). Na viragem do milénio, Larson (2000) chama a atenção para as altas taxas de aborrecimento, alienação e apatia por parte dos jovens, e para a necessidade de promover a iniciativa. Neste contexto, e tendo em vista o bemestar e o desenvolvimento dos jovens, o autor destaca a importância das oportunidades de participação dos jovens em atividades estruturadas e de participação livre.

Também conhecidas como atividades organizadas (Badura et al., 2016; Vandell et al., 2015), estas atividades distinguem-se de outras atividades na medida em que incluem: (a) estrutura e supervisão de adultos; (b) participação voluntária e regular capaz de permitir aos jovens aprofundarem os seus interesses; (c) dimensão de experiência prática; (d) interação entre pares; e (e) exigência do ponto de vista da motivação intrínseca, da concentração e do esforço para objetivos não imediatos (Barber et al., 2014; Larson, 2000; Vandell et al., 2015). Neste 
universo de atividades destacam-se, em primeiro lugar, as atividades extracurriculares de caráter desportivo, artístico ou vocacional, e cujo objetivo principal, para além do universo da escola e das competências académicas, é complementar o desenvolvimento da criança e dos jovens (Vandell et al., 2015). Em segundo lugar destacam-se as atividades orientadas para a participação e o serviço à comunidade (Barber et al., 2015), apresentadas como atividades de solidariedade de caráter voluntário.

No que diz respeito ao BEP, a importância da participação nestas atividades organizadas, de lazer ou caracterizadas pelo desafio e significado para os jovens, é confirmada pela associação com o bem-estar (Almeida, Pereira \& Fernandes, 2018; Freire et al., 2013). Alguns estudos na esteira da eudaimonia de Aristóteles, olham para a participação dos jovens nestas atividades, valorizando, além do impacto na dimensão Domínio do meio (Ryff, 2013), as oportunidades que os adolescentes encontram para, em relação com os outros, se conhecerem melhor a si mesmos, descobrirem o seu potencial pessoal, capacidades e talentos, explorarem identidades e, por outro lado, expressarem essas descobertas em contextos sociais concretos. Neste sentido, os estudos destacam atividades como as artes performativas, os desportos, a participação em grupos religiosos ou altruístas, por oposição a outras atividades de caráter mais passivo ou de entretenimento como ver televisão, atividades de risco como beber álcool, ou tempo descrito como passado com os amigos (Coatsworth \& Sharp, 2013).

No âmbito do Positive Youth Development a importância para os jovens da participação em atividades organizadas é confirmada pela associação a resultados como maior autoestima, sentimento de controlo da vida, maiores aspirações educativas e de realização pessoal, rendimento escolar, e menor frequência de comportamentos de risco (Agans et al., 2015; Barber et al., 2014; Larson, 2000; McCabe, Modecki \& Barber, 2016). No que diz respeito aos RD, um estudo com 99.462 jovens norte-americanos defende a relação positiva entre os RD e a participação em programas e atividades juvenis, a participação na comunidade religiosa, atividades de voluntariado e atividades criativas (Benson et al., 2011).

Procurando contribuir para o conhecimento em torno da participação em atividades organizadas, este estudo tem dois objetivos principais. O primeiro é caracterizar a amostra do ponto de vista da participação em atividades extracurriculares, em atividades de solidariedade e a sua regularidade, bem como da participação em atividades criativas ou religiosas (dimensão "Uso positivo do tempo" dos RD-Externos). O segundo objetivo é analisar a relação do bemestar psicológico (BEP) e dos recursos de desenvolvimento (RD) externos e internos com a participação em atividades extracurriculares, a participação em atividades de solidariedade e a regularidade da participação nas atividades de solidariedade. Neste sentido, a apresentação dos 
resultados e a sua discussão organiza-se em torno de três questões de estudo: (Q1) Como se distribuem os alunos pela participação em atividades extracurriculares e pela participação em atividades de solidariedade e a sua regularidade? (Q2) Quais as diferenças na participação em atividades criativas e em atividades religiosas em função do ano de escolaridade? (Q3) Como se relacionam o BEP e os RD, incluindo os RD-Externos e os RD-Internos, com a participação em atividades extracurriculares e a participação em atividades de solidariedade e a sua regularidade?

O presente estudo possui como particularidade a utilização de uma amostra constituída exclusivamente por alunos de uma escola particular de Lisboa (Portugal), o que permite pressupor um estatuto socioeconómico médio/ alto dos alunos. O estatuto socioeconómico surge em vários estudos, como variável positivamente relacionada com o BEP (Carvalho \& Veiga, 2020; Fernandes, 2007), com os RD (Benson \& Scales, 2014; Carvalho \& Veiga, 2020) e com o próprio envolvimento em atividades extracurriculares, marcado por fortes assimetrias (Meier, Hartmann \& Larson, 2018; Veiga, 2013; Veiga et al., 2019) e seletividade, com vantagem para os jovens com recursos socioeconómicos e culturais mais elevados, maior apoio parental e maior apoio escolar (Larson, 2000). Contudo a investigação mostra que o rendimento não é garante de bem-estar nem de crescimento humano (Ryff, 2018b); mostra também que a participação em atividades organizadas por parte de jovens com estatuto socioeconómico mais elevado não é isenta de fragilidades que podem inibir os benefícios da própria participação (Randall et al., 2016). Porque todas as realidades contextuais e pessoais possuem forças e fragilidades específicas que é preciso compreender, a amostra deste estudo é um convite a ir além do preconceito para identificar as exigências e desafios da realidade destes jovens.

\section{Método}

\section{Participantes}

A amostra do estudo é composta por 201 alunos de uma escola particular de Lisboa, entre os 10 e os 18 anos $(M=12,85)$, do sexo feminino (42,8\%) e do sexo masculino $(57,2 \%)$, e inclui os alunos do último ano de cada ciclo de estudos, isto é do $6^{\circ}$ ano $(52,2 \%)$, do $9^{\circ}$ ano $(31,8 \%)$ e do $11^{\circ}$ ano $(15,9 \%)$ em substituição do $12^{\circ}$ pelas especificidades associadas a este último ano.

\section{Instrumentos}

Para avaliar o BEP foi utilizada a EBEP-Escala de Bem-estar Psicológico, desenvolvida por Carol Ryff (1989). Neste estudo é usada a EBEP-Adolescentes, cuja 
adaptação à realidade portuguesa resulta do trabalho de Helder Fernandes (2007). A escala é formada por 30 itens que avaliam o BEP Global e cada umas das 6 dimensões do BEP: (a) Autonomia, (b) Domínio do meio, (c) Crescimento pessoal, (d) Relações positivas com os outros, (e) Objetivos na vida, (f) Aceitação de si. A resposta a cada item é feita através de uma escala tipo likert de 5 pontos ( 1 - discordo totalmente e 5 -discordo plenamente). Na sequência do estudo de validade interna optou-se pela utilização de uma versão reduzida da escala com 19 itens e apenas 5 dimensões (foi excluída a dimensão Domínio do Meio), com propriedades psicométricas adequadas à investigação.

Para avaliar os RD foi utilizado o DAP - Developmental Assets Profile, desenvolvido pelo Search Institute com o objetivo de superar as limitações de instrumentos anteriores e avaliar os RDs. O DAP é composto por 58 itens e duas subescalas: (a) Recursos de Desenvolvimento Externos (RD-Externos), com quatro dimensões e 26 itens, e (b) Recursos de Desenvolvimento Internos (RD-Internos) também com 4 dimensões e 32 itens. A resposta a cada item é feita através de uma escala de tipo likert de 4 pontos entre 0 (nunca ou raramente) e 3 (completamente ou quase sempre). No âmbito desta investigação procedeu-se à adaptação do instrumento à realidade portuguesa e optou-se, face a algumas fragilidades psicométricas, pela utilização de uma versão reduzida de 33 itens (14 itens na escala dos RD-Externos e 19 itens na escala dos RD-Internos), com qualidades psicométricas adequadas à investigação (Carvalho \& Veiga, 2017).

A avaliação da participação nas atividades foi feita a partir de três questões. A primeira questão avalia a participação em atividades extracurriculares ("Fora do tempo de aulas participas noutras atividades que gostas?"); a segunda questão avalia a participação em atividades solidárias ("Participas em atividades de solidariedade de carácter voluntário, isto é, por tua vontade?"). Para estas primeiras questões a resposta é apenas afirmativa ou negativa (Vandell et al., 2015; McCabe et al., 2016). A formulação da segunda questão procura distinguir entre a participação em atividades de carácter solidário realizadas por vontade própria, e outras atividades no âmbito do voluntariado, cujo enquadramento conceptual e etário seria diferente. A terceira questão, apenas para os alunos que afirmam participar em atividades de solidariedade, avalia a regularidade desta participação através de três opções: 1 ou 2 vezes por ano; 1 ou 2 vezes por mês; 1 ou 2 vezes por semana.

Na caracterização da participação em atividades é ainda considerada a dimensão "Uso positivo do Tempo", da subescala RD-Externos, que inclui os itens "Estou envolvido(a) num grupo religioso ou em atividades religiosas" (Item 31) e "Estou envolvido(a) em atividades criativas, tais como música, teatro ou arte" (Item 40). 


\section{Procedimentos}

Após a autorização dos autores dos instrumentos e da direção da escola, apresentou-se o estudo aos encarregados de educação e aos alunos. O consentimento informado foi assegurado através de uma autorização escrita dos encarregados de educação e da apresentação do estudo aos alunos. Fez parte dos procedimentos a realização de um estudo-piloto prévio com alunos do $7^{\circ}$ ano. A recolha dos dados foi feita na escola, com a presença do investigador, através de um questionário online (Google docs). O tratamento estatístico foi feito com recurso ao SPSS (20).

\section{Resultados}

A primeira questão de estudo (Q1) analisa a distribuição dos alunos pela participação em atividades extracurriculares e pela participação em atividades de solidariedade e a sua regularidade. Na Tabela 1 é possível ver que $81 \%$ dos alunos afirmam participar em atividades extracurriculares, e que $67,7 \%$ dos alunos afirmam participar em atividades de solidariedade de caráter voluntário. $\mathrm{O}$ número de alunos que afirmam participar em atividades extracurriculares diminui do $6^{\circ}$ para o $9^{\circ}$ e do $9^{\circ}$ para o $11^{\circ}$ ano. Uma diminuição, que apesar de mais suave, também se verifica nos alunos que afirmam participar em atividades de solidariedade. Contudo, é nos alunos mais velhos do $11^{\circ}$ ano, que encontramos a percentagem mais elevada de alunos que participa em atividades de solidariedade com maior regularidade (1 ou 2 vezes por semana).

Tabela 1 - Distribuição da amostra em função da participação das atividades (\%)

\begin{tabular}{|c|c|c|c|c|c|}
\hline \multirow{3}{*}{$\begin{array}{l} \\
\text { Atividades } \\
\text { extracurriculares }\end{array}$} & & $6^{\circ}$ Ano & $9^{\circ}$ Ano & $11^{\circ}$ Ano & Total \\
\hline & Sim & 85.7 & 76.6 & 71.9 & 80,6 \\
\hline & Não & 14.3 & 23.4 & 28.1 & 19,4 \\
\hline \multirow{4}{*}{$\begin{array}{l}\text { Atividades de } \\
\text { solidariedade }\end{array}$} & Não & 31.4 & 34.4 & 37.5 & 33,3 \\
\hline & Sim, anualmente & 53.3 & 56.3 & 28.1 & 50,2 \\
\hline & Sim, mensalmente & 7.6 & 6.3 & 3.1 & 6,5 \\
\hline & Sim, semanalmente & 7.6 & 3.1 & 31.3 & 10,0 \\
\hline
\end{tabular}

A segunda questão de estudo (Q2) analisa as diferenças em relação à dimensão "Uso positivo do tempo" entre os três grupos independentes de alunos do $6^{\circ}, 9^{\circ}$ e $11^{\circ}$ ano de escolaridade. Os valores de participação nestas atividades não possuem a expressividade encontrada na participação nas atividades extracurriculares ou de solidariedade, sendo que apenas $50,2 \%$ dos alunos referem a elevada ocorrência de envolvimento em "atividades criativas, tais como música, teatro ou arte" (item 40) e apenas 39,8\% dos alunos referem a elevada ocorrência do envolvimento "num grupo religioso ou em atividades religiosas" (item 
31). A análise diferencial entre os três grupos independentes de alunos do $6^{\circ}, 9^{\circ}$ e $11^{\circ}$ ano em relação à dimensão "Uso positivo do tempo", revelou diferenças estatisticamente significativas entre o $6^{\circ}$ e o $9^{\circ}$ ano $(T=4,526 ; p=.000)$. mas não com o $11^{\circ}$ ano, onde, como se pode ver na Figura 1, existe um aumento que, no caso do envolvimento em grupos ou atividades religiosas, ultrapassa os valores reportados pelos alunos do $6^{\circ}$ ano.

Figura 1. Frequência elevada nos itens da dimensão "Uso Positivo do Tempo"

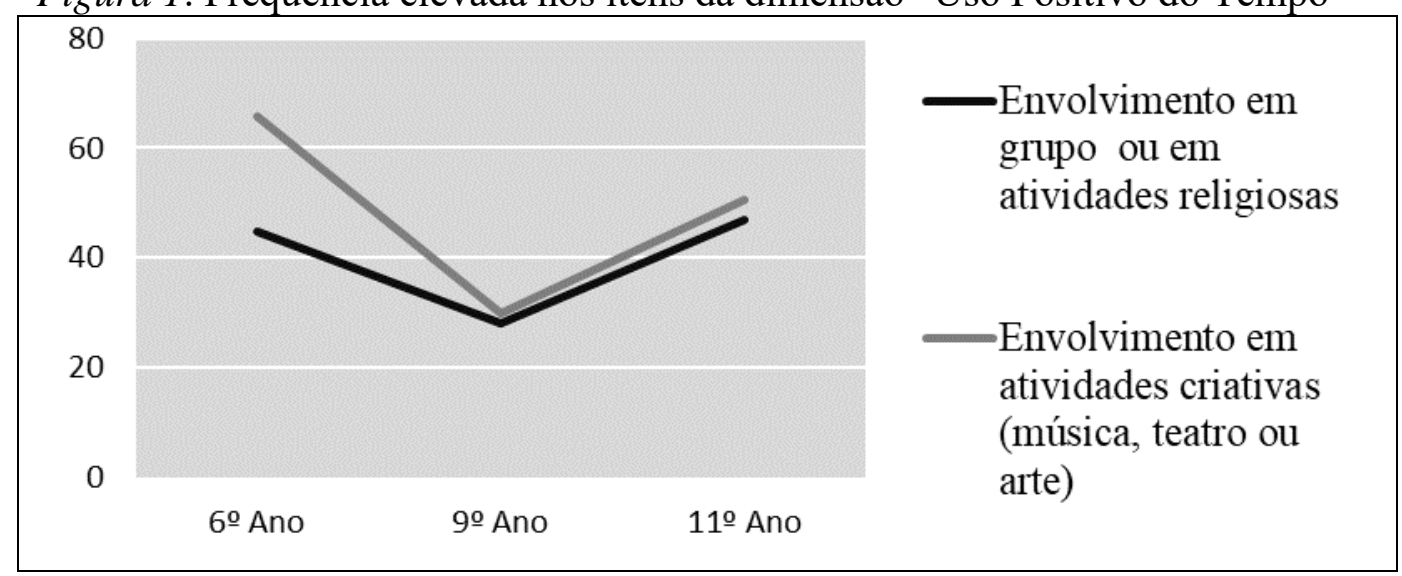

A terceira questão de estudo (Q3) analisa as correlações do BEP, dos RD-Externos, dos RD-Internos e das dimensões respetivas, com a participação nas atividades extracurriculares, nas atividades de solidariedade e a regularidade no caso das atividades de solidariedade. No âmbito do BEP, como mostra a Tabela 2, a participação nas atividades extracurriculares está correlacionada de forma estatisticamente significativa apenas com a dimensão "Aceitação de si" e com o item da dimensão "Relações positivas" (não especificado na tabela) "Sinto que tiro imenso partido das minhas amizades" (item 22). Por sua vez, a participação nas atividades de solidariedade está correlacionada de forma estatisticamente significativa com o BEP Global e as dimensões "Crescimento pessoal" e "Objetivos de vida". Não foi encontrada nenhuma correlação entre as dimensões do BEP e a regularidade da participação em atividades de solidariedade. Também não foi encontrada nenhuma correlação entre as dimensões do BEP "Autonomia" e "Relações positivas com os outros" e a participação nos dois tipos de atividades.

Tabela 2 - Correlações entre o Bem-estar psicológico e a participação em atividades

\begin{tabular}{|c|c|c|c|c|}
\hline & $\begin{array}{l}\text { Ativ. } \\
\text { extracurriculares }\end{array}$ & $\begin{array}{l}\text { Ativ. de } \\
\text { solidariedade }\end{array}$ & & $\begin{array}{l}\text { Regularidade das } \\
\text { ativ. solidariedade }\end{array}$ \\
\hline Crescimento pessoal & & ns & $.199^{* *}$ & \\
\hline Objetivos de vida & & ns & $.165^{*}$ & \\
\hline Aceitação de si & .15 & & ns & \\
\hline BEP Global & & ns & $.186^{* *}$ & \\
\hline
\end{tabular}

Legenda: $* p<0.05 ; * * p<0.01$; ns - não significativo 
No âmbito dos RD, como mostra a Tabela 3, a participação nas atividades extracurriculares, nas atividades de solidariedade e a regularidade da participação no caso das atividades de solidariedade apresentam correlações estatisticamente significativas com o Total dos RD, o total dos RD-Externos e o total dos RD-Internos. Contudo, existem algumas diferenças.

Tabela 3 - Correlações entre os Recursos de Desenvolvimento e a participação em atividades

\begin{tabular}{|c|c|c|c|}
\hline & $\begin{array}{l}\text { Ativ. A } \\
\text { extracurricul sc } \\
\text { ares }\end{array}$ & $\begin{array}{ll}\text { de } \\
\text { riedad }\end{array}$ & $\begin{array}{l}\text { Regularidade } \\
\text { das ativ. } \\
\text { solidariedade }\end{array}$ \\
\hline Total RD-Externos &, $169^{*}$ &, $\mathbf{2 2 3}^{* *}$ & ns \\
\hline Suporte & ns &, $147^{*}$ & $\overline{\mathrm{ns}}$ \\
\hline 13. Peço conselhos aos meus pais. &, $172^{*}$ & $\mathrm{~ns}$ & $\mathrm{~ns}$ \\
\hline Limites e expectativas & ns & $\mathbf{n s}$ & $\overline{\mathrm{ns}}$ \\
\hline $\begin{array}{l}\text { 50. Tenho professores que me encorajam a } \\
\text { desenvolver-me e a ir mais longe. }\end{array}$ & ns & , $163^{*}$ & ns \\
\hline $\begin{array}{l}\text { 57. Estou numa escola que aplica as regras de } \\
\text { forma justa. }\end{array}$ &, $142^{*}$ & ns & ns \\
\hline Uso positivo do tempo & ,192** & $\mathbf{2 8 0}^{* * *}$ & $\overline{\text { ns }}$ \\
\hline $\begin{array}{l}\text { 31. Estou envolvido(a) num grupo religioso ou } \\
\text { em atividades religiosas. }\end{array}$ & ns &, $247^{* *}$ &, $167^{*}$ \\
\hline $\begin{array}{l}\text { 40. Estou envolvido(a) em atividades criativas, } \\
\text { tais como música, teatro ou arte. }\end{array}$ &, $267^{* *}$ & ,149* & $\mathrm{ns}$ \\
\hline Total RD-Internos & ,180* &, $244^{* * *}$ & ,153* \\
\hline Compromisso com aprendizagem & ,194** & ,177* & \\
\hline 7. Gosto da escola. & ns & $238^{* *}$ & ns \\
\hline 8. Faço os meus trabalhos de casa. & $243^{* *}$ & ns & ns \\
\hline $\begin{array}{l}\text { 26. Estou empenhado(a) ativamente em } \\
\text { aprender coisas novas. }\end{array}$ &, $228^{* *}$ &, $184^{* *}$ &, $185^{* *}$ \\
\hline Competências sociais & ns &, $214^{* *}$ & ,146* \\
\hline 6. Crio amizades com outras pessoas. & ns & $225^{* *}$ &, $184^{* *}$ \\
\hline $\begin{array}{l}\text { 11. Exprimo os meus sentimentos de forma } \\
\text { apropriada. }\end{array}$ & ns & , $166^{*}$ &, $143^{*}$ \\
\hline Identidade positiva & ,184** & , 178* & ,162* \\
\hline 3. Sinto-me bem em relação a mim próprio(a). & ,149* & ns & \\
\hline 14. Lido com a frustração de forma positiva. & ns & ns & $155^{*}$ \\
\hline $\begin{array}{l}\text { 15. Arranjo formas boas de lidar com os } \\
\text { desafios. }\end{array}$ &, $202^{* *}$ & ,179* &, $162^{*}$ \\
\hline $\begin{array}{l}\text { 27. Estou a desenvolver um sentido para a } \\
\text { minha vida. }\end{array}$ & ns &, $146^{*}$ & ns \\
\hline Total RD & $194^{* *}$ & $259^{* *}$ & $\mathrm{~ns}$ \\
\hline
\end{tabular}

Legenda: ${ }^{*} p<0.05 ; * * p<0.01 ;$ ns - não significativo

No que diz respeito aos RD-Externos, destaca-se a correlação estatisticamente significativa da participação nos dois tipos de atividades com o "Uso positivo do tempo". Há contudo nesta dimensão algumas especificidades, uma vez que que a participação em atividades 
extracurriculares apenas está relacionada de forma estatisticamente significativa com o envolvimento em atividades criativas (item 40), enquanto a participação em atividades de solidariedade e a sua regularidade, além da correlação estatisticamente significativa com o envolvimento nas atividades criativas (item 40), está correlacionada de forma estatisticamente significativa com o envolvimento em grupos ou atividades religiosas (Item 31). Ainda ao nível dos RD-Externos existe uma correlação estatisticamente significativa entre a participação em atividades de solidariedade e a dimensão "suporte" por parte dos pais e outros adultos.

No âmbito dos RD-Internos, enquanto a participação nos dois tipos de atividades está correlacionada de forma estatisticamente significativa com as dimensões "Compromisso com a aprendizagem" e a "Identidade positiva", nesta última dimensão, além da correlação comum à participação nos dois tipos de atividades com a capacidade de encontrar formas boas de lidar com os desafios (Item 15), verifica-se que: (a) a participação em atividades extracurriculares está correlacionada de forma estatisticamente significativa com o sentir-se bem em relação a si mesmo (Item 3); (b) a participação em atividades de solidariedade está correlacionada de forma estatisticamente significativa com o desenvolvimento de um sentido para a vida (item 27); (c) a regularidade da participação em atividades de solidariedade está correlacionada com a capacidade de lidar com a frustração de forma positiva (item 14). Por último, ainda no âmbito dos RD-Internos destaca-se a correlação estatisticamente significativa entre a participação em atividades de solidariedade e a dimensão "Competências sociais".

\section{Discussão dos resultados}

$\mathrm{Na}$ primeira questão de estudo (Q1), que analisa a distribuição dos alunos pela participação nas atividades, destacam-se dois aspetos. O primeiro é o elevado número de alunos que afirmam participar em atividades extracurriculares $(80,6 \%)$ e de solidariedade $(66,7 \%)$. Esta realidade, não consistente com outros estudos com amostras portuguesas que refletem a baixa participação de adolescentes em atividades desportivas (e.g. Almeida et al., 2018; Marques, Peralta, Loureiro, Loureiro, Naia \& Matos, 2019), pode estar associada à especificidade da amostra, composta exclusivamente por alunos de uma escola particular. A hipótese é consistente com estudos que mostram que as oportunidades de participação em atividades organizadas são seletivas e marcadas pelo estatuto socioeconómico (Meier, et al., 2018; Vandell et al., 2015; Veiga et al.,2019). Esta realidade, contudo, não é linear. Alguns estudos sobre a participação em atividades organizadas de crianças e jovens de famílias com estatuto socioeconómico elevado sugerem que, apesar dos valores mais altos de participação, existem algumas questões, como o elevado número de horas que as crianças passam em 
atividades extracurriculares ou a motivação extrínseca (com efeitos negativos quando assenta exclusivamente na pressão dos pais), que condicionam os benefícios dessa participação para o bem-estar e desenvolvimento das crianças e adolescentes (Randall et al., 2016).

O segundo aspeto a destacar, nesta primeira questão de estudo, é a diminuição da participação nos dois tipos de atividades ao longo da adolescência, o que é consistente com outros estudos que referem a diminuição da atividade física e da participação desportiva ao longo da adolescência (Marques et al., 2019), e a diminuição da regularidade e da variedade das actividades ao longo da adolescência (Badura et al., 2016; Meier et al., 2018). Uma primeira explicação pode estar relacionada com a crescente pressão académica que tende a acentuar-se no secundário (Fernandes, 2007). Uma segunda razão pode estar associada ao paradoxo de que, à medida que os adolescentes crescem, revelando uma maior vontade e capacidade para assumirem as suas decisões, bem como uma maior autonomia face às decisões dos pais, de outros adultos e da escola, existirem menos opções disponíveis de participação em atividades organizadas (Larson, 2000). Uma terceira explicação, talvez associada à maior autonomia e desejo dos adolescentes para fazerem as suas próprias escolhas, chama a atenção para uma mudança no perfil de participação em atividades ao longo da escolaridade, com maior predomínio de opções não desportivas (Meier, et al., 2018) e de uma maior especialização na escolha das atividades (Vandell et al., 2015). Talvez esta mudança de perfil justifique a maior regularidade da participação em atividades de solidariedade nos alunos mais velhos (31,3\% dos alunos do $11^{\circ}$ ano afirma participar semanalmente em atividades de solidariedade).

Os resultados encontrados para a segunda questão de estudo (Q2), que analisa as diferenças na dimensão dos RD-Externos "Uso positivo do tempo" em função do ano de escolaridade, corroboram os dados apresentados na primeira questão no que diz respeito à acentuada diminuição do $6^{\circ}$ para o $9^{\circ}$ ano, destacando-se contudo, o aumento no $11^{\circ}$ ano do envolvimento em grupos ou atividades religiosas (Item 40), cujo valor supera o do $6^{\circ}$ ano. Exigindo maior aprofundamento teórico e empírico, os resultados são consistentes com o facto de as escolhas dos adolescentes mais velhos tenderem a ser intrinsecamente mais motivadas, autónomas e orientadas para os seus interesses, necessidades e para os desafios do próprio desenvolvimento (Larson, 2000; Vandell et al., 2015).

No que diz respeito à terceira questão de estudo (Q3), cujo objetivo é analisar a relação do BEP e dos RD com a participação nas atividades e a regularidade da participação nas atividades de solidariedade, verifica-se a correlação estatisticamente significativa do BEP Global apenas com a participação em atividades de solidariedade, e a correlação estatisticamente significativa do total dos RD, do total dos RD-Externos e o total dos RD- 
Internos com a participação em atividades extracurriculares, a participação em atividades de solidariedade e a maior regularidade da participação nas atividades de solidariedade. Estes resultados corroboram a importância atribuída às oportunidades de participação em atividades comunitárias, extracurriculares e voluntárias no bem-estar (Almeida et al., 2018; Barber et al., 2014; Freire et al., 2013) e no desenvolvimento juvenil positivo (Agans et al., 2015; Benson, et al., 2006; Larson 2000, McCabe et al., 2016; Vandell et al., 2015; Veiga et al., 2019), mas também evidenciam diferenças da participação nos dois tipos de atividades, visíveis desde logo na correlação não significativa entre o BEP e as atividades extracurriculares. Por esta razão, segue-se a discussão das correlações com o BEP e os RD comuns à participação nos dois tipos de atividades, sendo discutidas em segundo lugar as correlações que as distinguem.

A análise correlacional com o BEP e os RD identificou três resultados comuns à participação nas atividades extracurriculares e à participação nas atividades de solidariedade. O primeiro é a correlação da participação nos dois tipos de atividades com a dimensão dos RDInternos "Compromisso com a aprendizagem". É uma correlação consistente com outros estudos que defendem a associação entre a participação em atividades organizadas e uma maior satisfação e envolvimento na escola, rendimento e aspirações académicas (Badura et al., 2016; Barber et al., 2014; Vandell et al. 2015). Talvez a explicação esteja na possibilidade de a participação em atividades organizadas promover forças e recursos pessoais que se refletem na relação com a escola e nos resultados escolares (Badura et al., 2016). Outros autores complementam a ideia, defendendo que a participação em atividades organizadas pode facilitar a identificação emocional e social com valores e atitudes valorizadas no contexto escolar, bem como permitir a integração numa rede de pares que sejam referências académicas positivas (Eccles, Barber, Stone \& Hunt, 2003). É uma hipótese que levanta a questão dos pares, cuja influência, visível no âmbito dos valores (Ramiz \& Vakil, 2020) e em estudos sobre o envolvimento dos alunos na escola (e.g. Veiga, García, Reeve, Wentzel \& García, 2015), nem sempre é positiva, contribuindo para a qualidade das atividades e o tipo de impacto que a participação nestas poderá ter no bem-estar e desenvolvimento das crianças e jovens (McCabe et al., 2016; Vandell et al., 2015).

O segundo resultado comum, de certa forma associado à questão dos pares e à força dos laços forjados no contexto das atividades organizadas, é a correlação da participação nos dois tipos de atividades e as relações de amizade, que se expressa no caso da participação em atividades extracurriculares com o item do BEP "Tiro muito partido das minhas amizades" (dimensão "Relações positivas") e, no caso da participação em atividades de solidariedade e da sua regularidade, com a dimensão dos RD-Internos "Competências sociais", mais 
especificamente com a facilidade em criar amizades (Item 6) e a capacidade para expressar os sentimentos de forma apropriada (Item11). O terceiro resultado comum é a correlação entre a participação nos dois tipos de atividades e a capacidade de encontrar formas boas de lidar com os desafios (Item 15, dimensão dos RD-Internos "Identidade positiva"). Estes dois últimos resultados, comuns à participação tanto em atividades extracurriculares como em atividades de solidariedade, corroboram os estudos que indicam como benefícios da participação em atividades organizadas, o treino de capacidades físicas, intelectuais e sociais, a pertença a um grupo social, a pertença a uma rede de suporte de pares, a proximidade de adultos de referência e a experiência em lidar com desafios (Barber et al., 2014; Benson, 2006; Eccles et al., 2003).

Apesar dos aspetos comuns à participação nos dois tipos de atividades os resultados parecem sugerir a existência, para a participação em atividades extracurriculares por um lado, e para a participação em atividades de solidariedade por outro, de dois perfis diferentes de correlações com aspetos do BEP e dos RD, o que vai ao encontro de outros estudos que acentuam o impacto distinto da participação em diferentes tipos de atividades (e.g. Vandell et al., 2015; Barber et al., 2014).

No âmbito do BEP a participação em atividades extracurriculares correlaciona-se apenas com a dimensão "Aceitação de si”, enfatizando a associação à autoestima, enquanto a participação em atividades solidárias se correlaciona com as dimensões "Crescimento pessoal", “Objetivos de vida" e BEP Global. Esta especificidade é reforçada pelas correlações entre a participação nas atividades e os itens da dimensão dos RD-Internos "Identidade positiva" onde a participação em atividades extracurriculares surge associada à autoestima ("Sinto-me bem em relação a mim próprio"), e a participação em atividades de solidariedade surge mais associada às dimensões eudaimónicas do sentido e da autorrealização ("Estou a desenvolver um sentido para a minha vida"). Os resultados vão ao encontro de outros estudos que defendem que diferentes atividades possuem diferentes impactos no bem-estar e no desenvolvimento das crianças e jovens, e que parte desse impacto se deve ao nível de expressividade pessoal da atividade (Barber et al., 2014). Talvez por esta razão, de forma consistente com os estudos revistos, a participação em atividades organizadas de caráter extracurricular apareçam mais associadas à autoestima (Barber et al., 2014), enquanto a participação em atividades de solidariedade, integrando o conjunto das atividades religiosas, altruístas ou de grupos organizados como os escuteiros, apresentem níveis mais elevados de expressividade e, por essa razão, melhores resultados ao nível das dimensões eudaimónicas do sentido, crescimento pessoal e autorrealização (Coatsworth \& Sharp, 2013). Outros estudos destacam as atividades de carácter comunitário ou de serviço prossocial pelo potencial de duplo sentido, isto é, os 
jovens ao envolverem-se na transformação dos seus contextos, são eles próprios transformados, de forma proporcional ao nível de compromisso, protagonismo e sentido de agência, mas também, de forma proporcional às oportunidades que tiverem para refletirem sobre a experiência que viveram (Benson et al., 2006).

Esta necessidade de estrutura, enquadramento e de reflexão no que diz respeito às atividades de caráter solidário e voluntário, pode justificar a importância dos pais e da escola na participação em atividades de solidariedade, expressa na correlação exclusiva da participação nas atividades de solidariedade com a dimensão dos RD-Externos "Suporte" e, na dimensão "Limites e expectativas", com o item associado ao encorajamento dos professores (Item 50).

Ainda distinguindo a participação num e noutro tipo de atividades, destaca-se a correlação exclusiva entre a participação em atividades de solidariedade e a maior regularidade desta e o envolvimento em grupos ou atividades religiosas (item 31 da dimensão dos RDExternos "Uso positivo do tempo"). A temática da religião e da espiritualidade está muito presente no estudo do BEP (Fernandes, 2007) e dos RD (Wagener, Furrow, King, Leffert \& Benson, 2003). Alguns estudos evidenciam a relação entre a participação numa comunidade religiosa e o envolvimento cívico, o serviço comunitário, o altruísmo e os compromissos prossociais (King \& Boyatzis 2015). Os resultados encontrados em torno da participação mais regular em atividades de solidariedade por parte dos alunos do $11^{\circ}$ ano são também consistentes com estudos que mostram a maior influência da variável religião em alunos mais velhos, onde diminui a influência e expectativas dos pais para uma ação que reflete uma adesão mais pessoal (Wagener et al., 2003).

Um último aspeto que distingue a participação nos dois tipos de atividades diz respeito à relação exclusiva entre a maior regularidade da participação em atividades de solidariedade e, no âmbito dos RD-Internos, a capacidade para lidar com a frustração de forma positiva (Item 14). Carecendo de maior aprofundamento fica como possibilidade o facto de o envolvimento em atividades de solidariedade com maior regularidade permitir aos adolescentes o confronto com desafios e adversidades que podem ser promotores de resiliência (Ryff, 2013).

\section{Considerações finais}

Em síntese, os resultados deste estudo permitem reiterar a importância das oportunidades de participação em atividades extracurriculares e de solidariedade no bem-estar e no desenvolvimento positivo das crianças e jovens. Destacam-se também os diferentes resultados encontrados para a participação em atividades extracurriculares, mais associados à 
autoestima, e para a participação em atividades de solidariedade, mais associados ao crescimento pessoal, à autorrealização e à resiliência. São resultados que contribuem para reforçar a necessidade de uma maior atenção em relação às oportunidades existentes nos contextos de desenvolvimento com destaque para escola (Ryff, 2018a), bem como, em relação à importância de investir em oportunidades de participação em diferentes tipos de atividades, permitindo apoiar e promover as várias dimensões essenciais ao bem-estar e ao desenvolvimento dos alunos ao longo da escolaridade. Alguns projetos em Portugal, nomeadamente na escola onde se realizou o estudo, têm procurado concretizar este desafio no sentido de oportunidades de participação em atividades estruturadas mais desafiantes e significativas (Carvalho, 2019; Carvalho e Veiga, 2020).

Para estudos futuros, uma vez que esta é uma área em que predominam os estudos norteamericanos, sugere-se um olhar geográfica e culturalmente mais alargado (Badura et al., 2016). De forma mais particular, face às opções do presente estudos, sugere-se a importância de estudos com amostras mais diversificadas, e uma melhor caracterização da participação ao nível da duração, impacto, variedade e tipo de atividades (Agans et al., 2015; Veiga et al., 2019).

\section{Nota:}

O presente estudo insere-se num outro mais amplo com o título "Bem-estar psicológico e recursos contextuais e pessoais em adolescentes de uma escola particular portuguesa" (dissertação de mestrado), realizada pelo primeiro autor com a orientação do segundo autor, Professor Doutor Feliciano H. Veiga (Carvalho, 2015).

\section{REFERÊNCIAS}

Agans, J. P., Champine R. B., Johnson, S. K., Erickson, K. \& Yalin, C. (2015). Promoting healthy lifestyles through youth activity participation: lessons from research In E. Bowers, G. Geldhof, S. Johnson, L. Hilliard, R. Hershberg, J. Lerner \& R. Lerner (Eds). Promoting positive youth development: Lessons from the 4-H study (pp. 137-158). New York: Springer.

Almeida, L. M., Pereira, H. P., \& Fernandes, H. M. (2018). Efeitos de diferentes tipos de prática desportiva no bem-estar psicológico de jovens estudantes do ensino profissional. Revista Iberoamericana de Psicología del Ejercicio y el Deporte, 13(1), 15-21.

Badura, P., Sigmund, E., Geckova, A. M., Sigmundova D., Sirucek, J., vanDijk, J. P., \& Reijneveld, S. A. (2016). Is participation in organized leisure-time activities associated with school performance in adolescence? PLos One, 11(4), 1-13. DOI:

https://doi.org/10.1371/journal.pone.0153276

Barber, B. L., Abbott, B. D., Neira, C. J. B., E., \& Eccles, J. S. (2014). Meaningful activity participation and positive youth development. In M. J. Furlong, R. Gilman, E. S. Huebner (Eds). Handbook of Positive Psychology in Schools (2nd Edition) (pp. pp.227-244). New York: Routledge. 
Benson, P. L, Scales, P. C., \& Syvertsen, A. K. (2011). The contribution of the developmental assets framework to positive youth development theory and practice. Advances in Child Development and Behavior, 41, 197-230.

Benson, P. L., \& Scales, P. C. (2014). Developmental Assets. In: R. J. R. Levesque (Ed.). Encyclopedia of Adolescence (pp. 667-683). New York: Springer.

Benson, P. L., Scales, P. C., Hamilton, S. F. \& Sesma, A. (2006). Positive youth development so far: core hypotheses and their implications for practice. Search Institute Insights \& Evidence, 3(1), 1-13.

Carvalho, N. A. (2015). Bem-estar psicológico e recursos contextuais e pessoais em adolescentes de uma escola particular portuguesa (Dissertação de mestrado orientada pelo Professor Doutor Feliciano Veiga). Lisboa: Instituto de Educação da Universidade de Lisboa. DOI: https://doi.org/10.13140/RG.2.2.22472.67844

Carvalho, N. A. (2019). Formação Humana no currículo: Um programa para fazer à medida. Sisyphus - Journal of education, 7(02), 65-91. DOI: https://doi.org/10.25749/sis.17260

Carvalho, N. A., \& Veiga, F. H. (2017). Recursos individuais e contextuais de desenvolvimento pessoal: Adaptação portuguesa do Developmental Assets Profile. Revista de Estudios e Investigación en Psicología y Educación, Vol. Extr. (14), 122-127. DOI: https://doi.org/10.17979/reipe.2017.0.14.2537

Carvalho, N.A., \& Veiga, F. H. (2020). Bem-estar psicológico e recursos de desenvolvimento: Caracterização em alunos adolescentes. Psicologia em Pesquisa, 14(2). DOI: http://dx.doi.org/10.34019/1982-1247.2020.v14.27503

Coatsworth, J. D. \& Sharp, E. H. (2013). Discovering positive lives and futures: Adolescent eudaimonic expression through activity involvement. In: A. S. Waterman (Ed.). The best within us: Positive psychology perspectives on eudaimonia (pp. 249-267). Washington, DC: American Psychological Association.

Eccles, J. S., Barber, B. L., Stone, M. R. \& Hunt, J. (2003). Extracurricular activities and adolescent development. Journal of Social Issues, 59(4), 865-889.

Fernandes, H. (2007). O bem-estar psicológico em adolescentes: uma abordagem centrada no florescimento humano (Tese doutoramento em psicologia). Vila Real: UTAD.

Freire, T., Zenhas, F., Tavares, D. \& Iglésias, C. (2013). Felicidade hedónica e eudaimónica: Um estudo com adolescentes portugueses. Análise Psicológica, 31(4), 329-342.

Gama, S. (2003). Diário de Sebastião da Gama: Pequena história da minha vida de professor. Sintra: Edições Arrábida.

King, P. E., \& Boyatzis, C. J. (2015). Religious and spiritual development. In R. M. Lerner (Eds). Handbook of Child Psychology and Developmental Science, Vol. 4 (pp. 975-1021). New Jersey: John Wiley \& Sons, Inc.

Larson, R. (2000). Toward a Psychology of Positive Youth Development. American Psychologist, 55(1), 70-183. DOI: https://doi.org/10.1037/0003-066X.55.1.170

Marques, A., Peralta, M., Loureiro, N., Loureiro, V. Naia, A., \& Matos, M. G. (2019). A atividade física dos adolescentes portugueses, resultados do estudo HBSC de 2018. Revista Psicologia da Criança e do Adolescente, 10(1), 129-137.

McCabe, K. O., Modecki, K. L., \& Barber, B. L. (2016). Participation in Organized Activities Protects Against Adolescents' Risky Substance Use, Even Beyond Development in Conscientiousness. Journal of Youth and Adolescence, 45, 2292-2306. DOI:

https://doi.org/10.1007/s10964-016-0454-x 
Meier, A., Hartmann, S., \& Larson R. (2018). A Quarter Century of Participation in SchoolBased Extracurricular Activities: Inequalities by Race, Class, Gender and Age? Journal of Youth and Adolescence, 47(6), 1299-1316. DOI: https://doi.org/10.1007/s10964-018-0838$\underline{\text { 1OECD }}$ (2018). The future of education and skills: Education 2030. Paris: The Author.

Ramiz, A. K., \& Vakil, C. R. (2020). Socio-psychological issues of changing values in adolescents-Youth in education. Práxis Educacional, 16(37), 578-590. https://doi.org/10.22481/praxisedu.v16i37.6406

Randall, E. T., Travers, L. V., Shapiro, J. B. \& Bohnert, A. (2016). Reasons for the " afterschool pressure cooker' in Affluent Communities: It's Not How Much Time, but Why. Journal of Child and Family Studies 25(5), 1559-1569. DOI: https://doi.org/10.1007/s10826$\underline{015-0330-4}$

Ryff, C. D. (1989). Happiness is everything, or is it? Explorations on the meaning of psychological well being. Journal of Personality and Social Psychology, 57, 1069-1081.

Ryff, C. D. (2013). Eudaimonic well-being and health: Mapping consequences of selfrealization. In: A. S. Waterman (Ed.). The best within us: Positive psychology perspectives on eudaimonia (pp. 77-98). Washington, DC: American Psychological Association.

Ryff, C. D. (2018a). Eudaimonic well-being: Highlights from 25 years of inquiry. In K. Shigemasu, S. Kuwano, T. Sato, \& T. Matsuzawa (Eds.), Diversity in harmony - Insights from psychology: Proceedings of the 31 st International Congress of Psychology (p. 375-395). John Wiley \& Sons Ltd. https://doi.org/10.1002/9781119362081.ch20

Ryff, C. D. (2018b). Well-being with soul: Science in pursuit of human potential. Perspectives on Psychological Science, 13(2), 242-248. DOI: https://doi.org/10.1177/1745691617699836

Scales, P. C., Roehlkepartain, E. C. \& Shramko, M. (2017). Aligning youth development theory, measurement, and practice across cultures and contexts: Lessons from use of the Developmental Assets Profile. Child Indicators Research, 10(4), 1145-1178. DOI: https://doi.org/10.1007/s12187-016-9395-X

Vandell, D. L., Larson, R. W., Mahoney, J. L. \& Watts, T. (2015). Children's Organized Activities. In R. M. Lerner (Eds). Handbook of Child Psychology and Developmental Science, Vol. 4 (pp. 305-344). New Jersey: John Wiley \& Sons, Inc. DOI: https://doi.org/10.1002/9781118963418.childpsy408

Veiga, F. (Org.) (2013). Psicologia da Educação: Teoria, Investigação e Aplicação Envolvimento dos Alunos na Escola. Lisboa: Climepsi.

Veiga, F. H., García, F., Reeve, J., Wentzel, K., \& García, Ó. (2015). When Adolescents with High Self-Concept Lose their Engagement in School. Revista de Psicodidáctica, 20(2), 305320. DOI: https://doi.org/10.1387/RevPsicodidact.12671

Veiga, F. H., García, Ó., \& García, F. (2019). Envolvimento dos Alunos na Escola: Conceptualização, Avaliação e Promoção. In F. H. Veiga (Coord.), Psicologia da Educação: Temas de Aprofundamento Científico para a Educação XXI (pp. 37-60). Lisboa: Climepsi.

Wagener L. M., Furrow J. L., King P. E., Leffert, N. \& Benson, P. (2003). Religious Involvement and Developmental Resources in Youth. Review of Religious Research, 44 (3), 271-284. DOI: https://doi.org/10.2307/3512387 


\section{SOBRE OS AUTORES:}

\section{Nuno Archer de Carvalho}

Mestrado, IE-ULisboa; Colégio Pedro Arrupe, Portugal; Doutoramento em Educação, especialidade Psicologia da Educação, IE-Ulisboa; narcher@campus.ul.pt

(iD https://orcid.org/0000-0001-6620-0804

\section{Feliciano Henriques Veiga}

Professor Catedrático, IE-Ulisboa; Instituto de Educação da Universidade de Lisboa, Portugal; Educação psicologia da educação; fhveiga@ie.ulisboa.pt

(iD https://orcid.org/0000-0002-2977-6238 